\title{
Imaging and minimally invasive endovascular therapy in the management of portal vein thrombosis
}

\author{
Susana G. Rodrigues, ${ }^{1}$ Martin H. Maurer, ${ }^{2}$ Iris Baumgartner, ${ }^{3}$ Andrea De Gottardi, ${ }^{1}$ \\ Annalisa Berzigotti ${ }^{1}$ \\ ${ }^{1}$ Swiss Liver Center, Hepatology, University Clinic for Visceral Surgery and Medicine, Inselspital, University of Bern, MEM F807, \\ Murtenstrasse 35, 3010 Bern, Switzerland \\ ${ }^{2}$ Department of Radiology, Inselspital, University of Bern, Bern, Switzerland \\ ${ }^{3}$ Clinic for Angiology, Swiss Cardiovascular Center, Inselspital, University of Bern, Bern, Switzerland
}

\begin{abstract}
Thrombosis of the portal venous system, although rare in the general population, is commonly diagnosed in patients with specific underlying conditions including prothrombotic diseases, cirrhosis, hepatobiliary malignancy, and intraabdominal inflammation. Recent improvements in imaging have played a fundamental role in increased detection of portal vein thrombosis (PVT), frequently reported in asymptomatic patients as an incidental finding. Minimally invasive, endovascular therapy is a medically rational option to achieve recanalization of the portal vein as an adjunct to conservative medical management. This review focuses on the advances in imaging modalities to diagnose, stage and follow-up PVT, and gives a short overview of the available endovascular techniques in this field.
\end{abstract}

Key words: Thrombus - Cavernous transformation-Cirrhosis-Thrombophilia-Portal hypertension-Ultrasound-Transjugular intrahepatic portosytemic shunt

The frequent and disseminated use of noninvasive liver imaging methods has led to an increased detection of thrombosis of the portal venous system. The thrombus fills the lumen of the portal (PV), splenic (SV), and superior and inferior mesenteric veins (SMV, IMV). It

Susana G. Rodrigues and Martin H. Maurer have equally contributed to this work.

Correspondence to: Annalisa Berzigotti; email: annalisa.berzigotti@ insel.ch can be anatomically diffuse or restricted to one vessel or to some of its branches. Portal vein thrombosis (PVT) refers to the partial or complete obstruction of portal venous flow by a luminal clot, or to the presence of a "cavernous transformation", constituted by hepatopetal collateral neovessels at the porta hepatis, that develop to bypass the obstructed segment of the portal venous system.

The prevalence of non-tumorous PVT in the general population has been estimated to be about $1 \%$ based on an autopsy series [1]. PVT is the second most common cause of portal hypertension in the developed world. The etiology of PVT varies [2,3] and, frequently, is linked to multiple concomitant factors [4]. Cirrhosis, hepatobiliary cancer, and other local factors, such as surgery and intraabdominal inflammation increase susceptibility to PVT [2]. In non-cirrhotic, non-malignant PVT (also termed extrahepatic portal vein obstruction-EHPVO) [5], systemic factors, i.e., inherited and acquired prothrombotic diseases or primary myeloproliferative disorders, are often found and play a major pathogenetic role $[6,7]$. Recently, abdominal obesity has been suggested as a possible additional risk factor [8].

Liver cirrhosis is currently recognized as a prothrombotic condition $[9,10]$. Among patients with cirrhosis, PVT is a more frequent event as compared to the general population, and its prevalence is between $10 \%$ and $25 \%$, being the highest observed in decompensated, Child C patients [11]. A large prospective study of patients with compensated Child A and B cirrhosis showed a 1 and 5-year cumulative incidence of PVT of $4.6 \%$ and $10.7 \%$, respectively [12]. This data is in line with the concept that PVT risk increases upon worsening of any of the components of Virchow's triad, i.e., stasis (here 
Table 1. New Anatomico-functional classification of portal vein thrombosis in cirrhosis. Adapted from Sarin et al. [16]

\begin{tabular}{l} 
Terminology to be used \\
\hline I-Site \\
Type 1: only trunk \\
Type 2: only branch \\
(2a): one branch \\
(2b): two branches \\
Type 3: Trunk and branches \\
II- Degree of portal vein occlusion \\
Occlusive: no visible flow in portal vein (PV) lumen \\
Nonocclusive: flow visible in PV lumen \\
IIIa-Duration \\
Recent: first detection in previously patent PV, dilated PV, hyperdense thrombus, absent, or limited collaterals \\
Chronic: previously diagnosed PVT, cavernous transformation of the PV, no hyperdense thrombus, signs of PH \\
IIIb-Presentation \\
Asymptomatic \\
Symptomatic: \\
If Recent Acute PVT (with or without bowel ischemia) \\
If Chronic Clinical manifestations of PH (variceal bleeding, ascites) \\
IV-Extent of PV system occlusion \\
Splenic vein \\
Mesenteric vein \\
Both
\end{tabular}

${ }^{\text {a }}$ refer to $\mathrm{CT}$ imaging

represented by decreased portal venous blood flow velocity) [13], hypercoagulability [10], and endothelial damage [14].

Clinicians may suspect PVT in the setting of abdominal pain, which can dominate the clinical features, particularly when thrombosis is recent and extending to the mesenteric venous system. In patients with chronic PVT, suspicion is often raised by presence of complications of portal hypertension $(\mathrm{PH})$ such as variceal bleeding, ascites, encephalopathy, and/or portal cholangiopathy (PC), featuring jaundice or cholangitis. In these cases, the differentiation from simple decompensation of cirrhosis is key. Nevertheless, PVT is frequently asymptomatic and incidentally identified on imaging. This raised awareness of healthcare professionals aligned with the notable developments of imaging technology has fueled growing knowledge in this complex setting. This review aims to provide a comprehensive outlook on the current role imaging plays in diagnosing and treating this entity.

\section{Classification}

From an imaging perspective, PVT is primarily defined based on structural characteristics such as location (PV, SMV, or SV), degree of occlusion, presence of cavernous transformation of the PV, signs of $\mathrm{PH}, \mathrm{PC}$ or signs of bowel ischemia. Recent classifications of PVT, such as the commonly used Yerdel's classification [15], have focused primarily on anatomical findings, ignoring the functional impact. The Baveno consensus conference on the management of portal hypertension is an interdisciplinary (hepatology, radiology, surgery, pediatrics) international expert meeting held every 5 years focusing on controver- sial and unclarified aspects in this field. Given the complexity of PVT, the expert panel opinion was to classify PVT according to: site, presentation/timing, underlying disease, and degree and extent of occlusion [5]. Whether thrombosis occurs in the setting of cirrhosis or in the absence of cirrhosis is relevant information that should be taken into consideration and PVT in the absence of cirrhosis should be defined as "EHPVO". A new structural and functional classification of PVT in cirrhotic patients has been recently proposed by the experts in PVT belonging to the Baveno VI (2015) consensus conference expert panel to uniformly report findings and to homogenize, stratify, and follow the natural history of this heterogeneous group of patients [16]. This defines the patient by the site, degree, presentation, and functional relevance of the thrombosis, i.e., acute or recent versus chronic with or without cavernous transformation of the $\mathrm{PV}$, and symptomatic with or without acute bowel ischemia versus asymptomatic (Table 1).

\section{Imaging in diagnosis, risk stratification, and planning of management of PVT: description of the available techniques}

Imaging is the cornerstone of the diagnosis of PVT. Ideally, an imaging method should inform on the presence or absence of thrombosis, and if PVT is diagnosed, the report should contain anatomical and functional pertinent information: thrombosis nature (thrombosis vs. neoplastic invasion; see "Differentiating thrombosis from tumorous invasion of the portal vein"), thrombus extension, estimation of timing/chronicity, presence or absence of a local factor, presence or absence of com- 


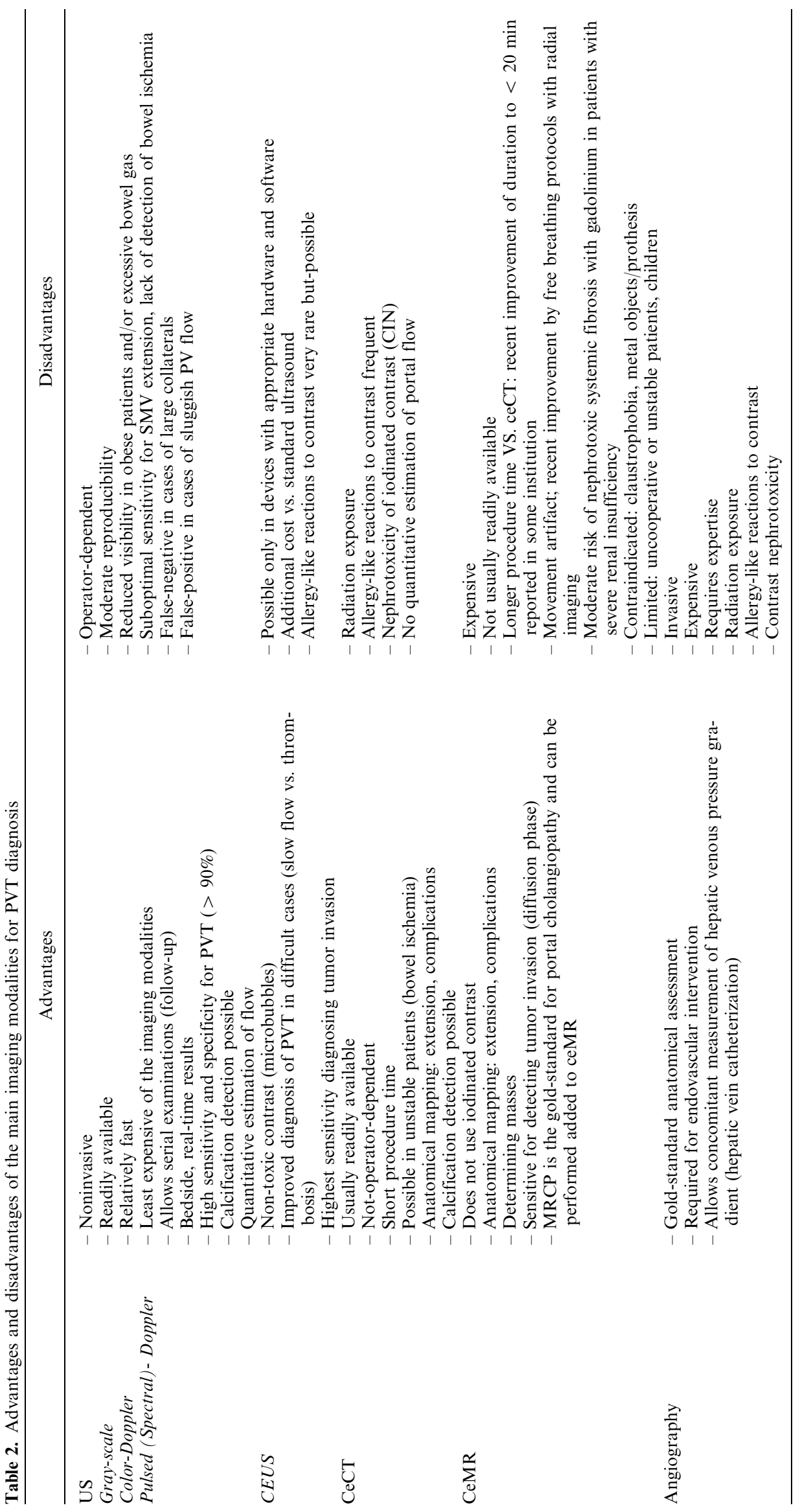


plications such as ascites, and congestion or ischemia of the bowel [7].

First level imaging should inform on the presence of absence of thrombosis. Ultrasound (gray-scale and Doppler imaging) is accurate to diagnose and exclude PVT and should be used as the first-line imaging method. [17, 18]. Contrast-enhanced (ce) imaging (ce-computed tomography, ceCT, or, if contraindicated, ce-magnetic resonance, ceMR) should be considered on admission in patients presenting with severe presentation, particularly insuspected mesenteric ischemia, or with known severe underlying disease (malignancy; history of intraabdominal inflammation/surgery, thrombophilia) or in whom ultrasound cannot be correctly applied (visualization limits) [5, 17].

Furthermore, ceCT or ceMR should be used in patients who have been diagnosed with PVT by ultrasonography to achieve a thorough characterization (anatomical staging, collateral mapping, detection of local factors, and complications). Presence of gas in the portal vein observed by any imaging technique should prompt a strong suspicion of intestinal ischemia. Table 2 shows the main advantages and disadvantages of each imaging method used in PVT diagnosis.

\section{Ultrasonography}

Gray scale (B-mode) ultrasonography (US) complemented by color-doppler ultrasonography (CDUS) is considered the first imaging examination to be performed because sensitivity and specificity values are above $90 \%$ [19]. It is well-tolerated, safe, inexpensive, readily available, repeatable and given its high negative predictive value, if CDUS confirms PV patency, then no further studies are necessary. The sensitivity of ultrasonography for diagnosis of PVT partly depends on the expertise of the operator, interpatient variability and stage of
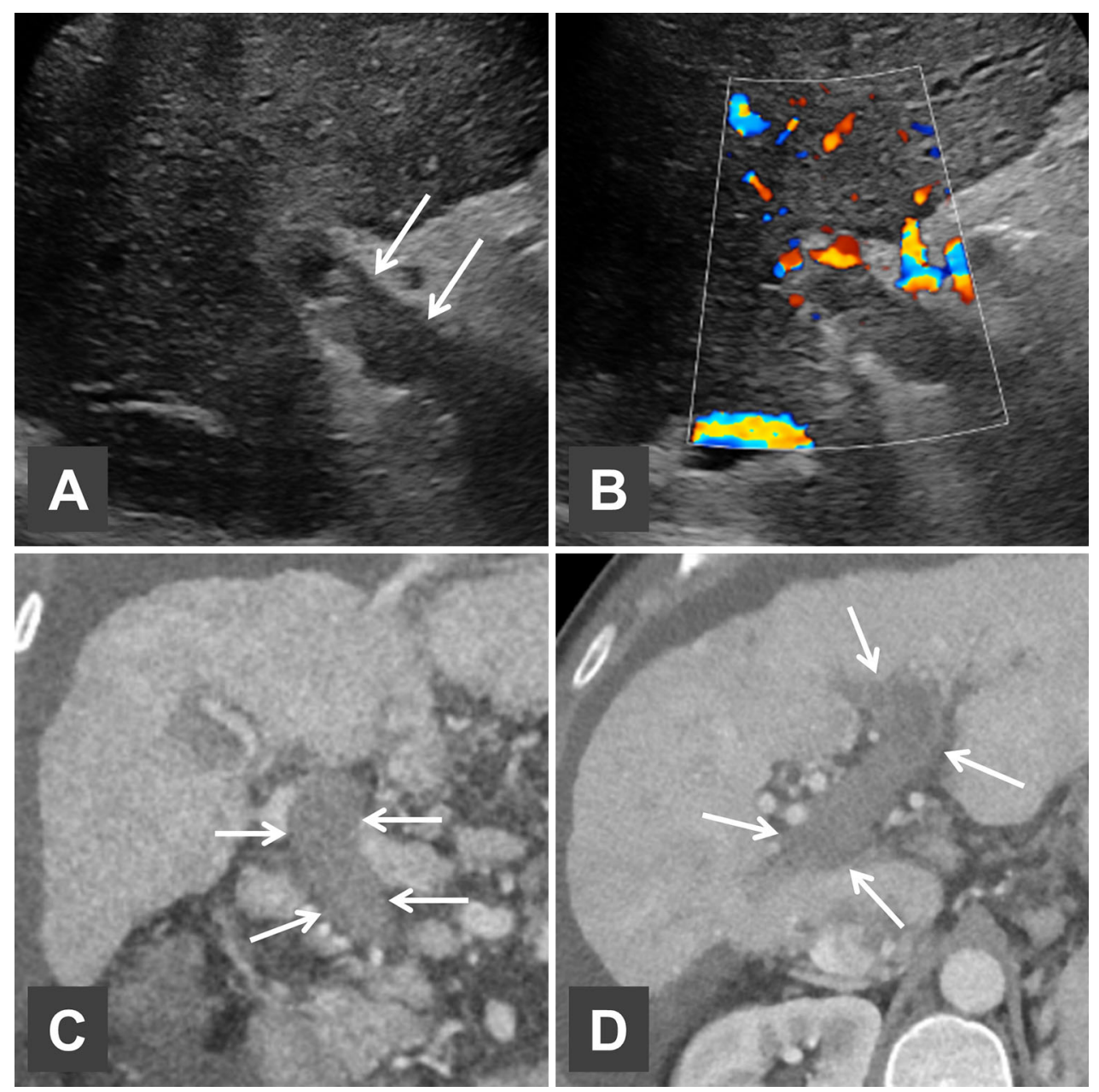

Fig. 1. Fifty seven years old male with a known Child B alcoholic cirrhosis of the liver who developed upper abdominal pain. An ultrasonography shows a complete acute occlusion of the main trunk of the portal vein (arrows, A) with only a few small collateralizing vessels in the Doppler ultra-

sonography (B). A CT scan of the same patient reveals hypodense thrombotic material completely occluding the main portal vein trunk (arrows in coronal view, C) as well as the right and the left intrahepatic portal venous branches (arrows in axial view, D). 

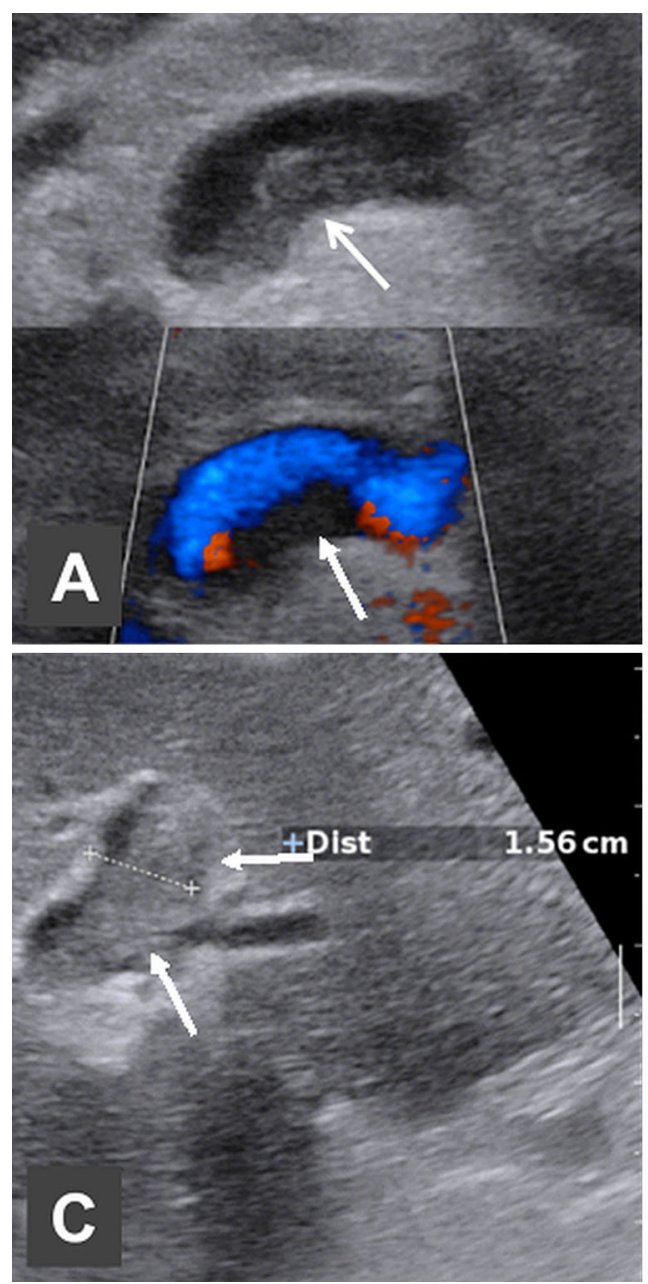

Fig. 2. Partial (mural) thrombosis of the right (A and $\mathbf{B}$ ) and left ( $\mathbf{B}$ and $\mathbf{C}$ ) intrahepatic portal vein. The thrombotic material is hyperechogenic in the B-mode image of the ultrasonography (arrow) while a partial perfusion of the vessel is remaining (A). A coronal CT view shows the hypodense thrombus in the

obstruction and has been reported to be $100 \%$ in Yerdel stages 3 and 4, i.e., complete thrombosis of the PV with involvement of the SMV [15]; ultrasound is however less sensitive if thrombosis is limited to SMV.

On gray scale ultrasonography, PVT can be seen as echogenic material within the PV lumen, occupying it completely (Fig. 1) or partially (Fig. 2). PV can appear dilated in the site of a complete and recent occlusion (Fig. 2). The echogenicity of the clot is variable from hypo-, iso- or hyperechoic, being a hypoechoic aspect more commonly observed in recent PVT. Both CDUS and pulsed (spectral) Doppler US can differentiate complete occlusive thrombosis from parietal incomplete thrombosis. Complete thrombosis is characterized by complete lack of color filling and Doppler signals. Thus Doppler techniques facilitate the US examination and improve its accuracy. These techniques are usually used in combination (Fig. 1).

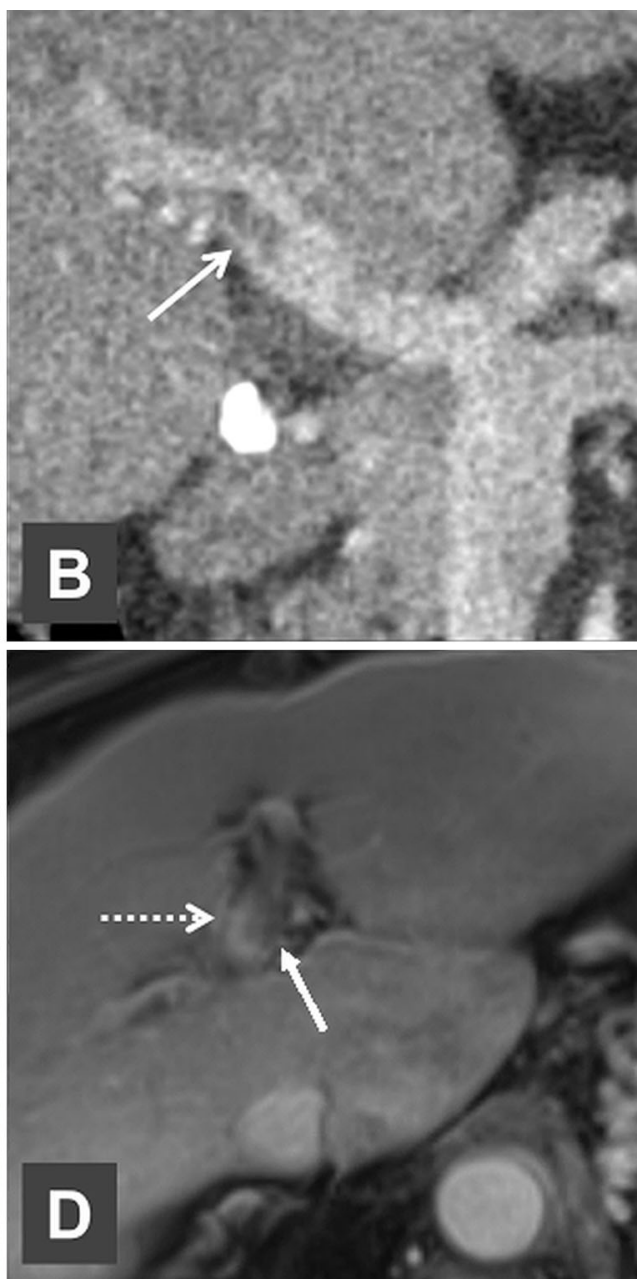

right portal vein (B). Partial thrombosis of the left intrahepatic vein in another patient: the diameter of the vessel is enlarged (measurement, C) and a contrast-enhanced magnetic resonance imaging (ceMRI) depicts a partial thrombosis (dotted arrow, D).

Alternatively, lack of a normal PV and observance of small tortuous vessels with hepatopetal flow lead to the diagnosis of cavernous transformation of the PV [19, 20] (Fig. 3). In patients with cavernous transformation of the PV or long-lasting EHPVO, morphological changes of the liver, compensatory hypertrophy of the hepatic artery and signs of portal hypertension (splenomegaly; porto-systemic collaterals) are frequent associated findings.

Endoscopic ultrasonography (endosonography) is both sensitive and specific for PVT. It can detect small and nonocclusive thrombi [21, 22]; however, this imaging modality is only used in second or third instance when diagnostic doubts persist after noninvasive imaging studies.

In the last decade, contrast-enhanced ultrasound (CEUS) has been introduced. The method is based on the intravenous injection of ultrasound contrast agents, 

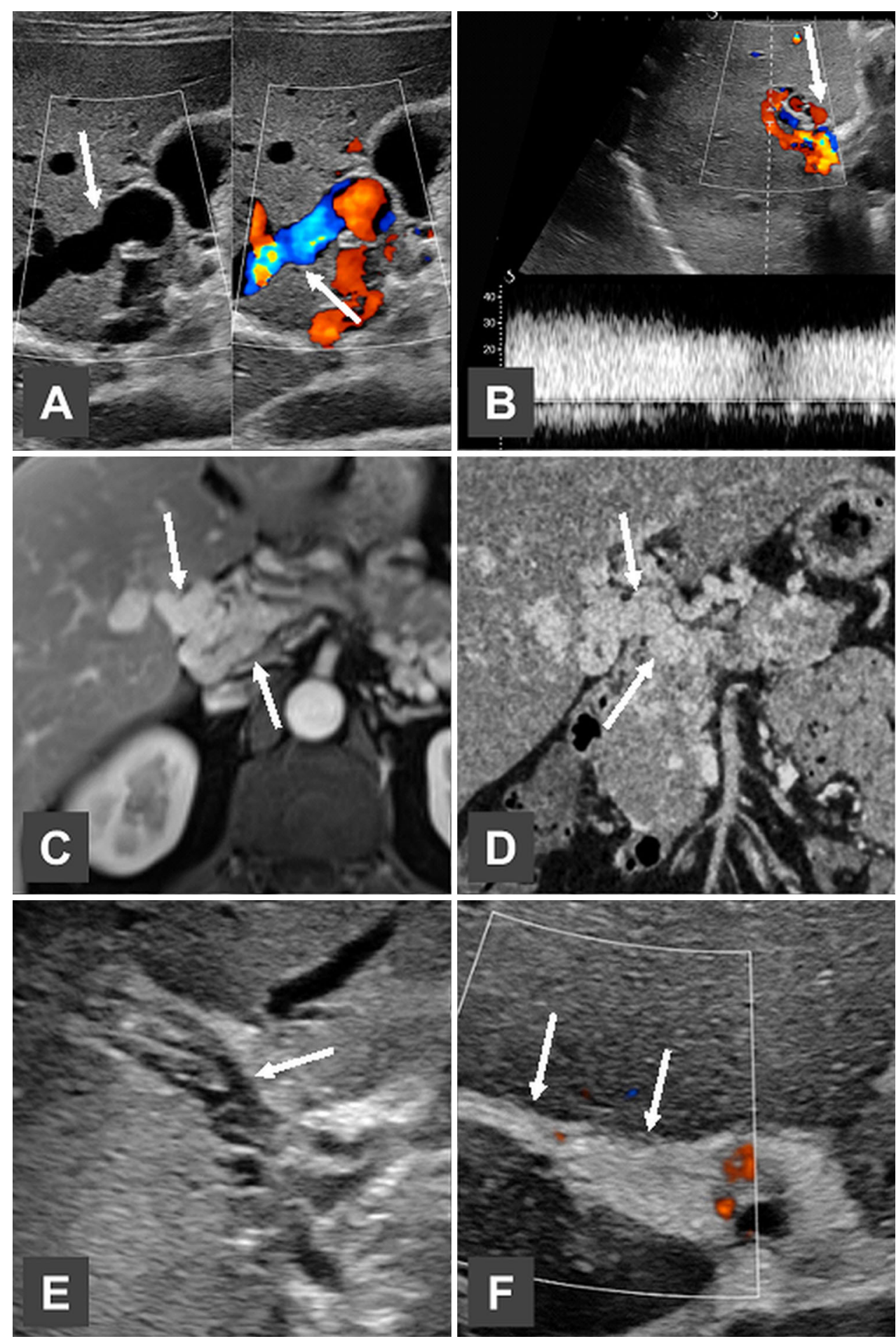

Fig. 3. Chronic complete portal vein thrombosis with a web of large collateral vessels along the contour of the portal vein (arrow) but with no remnant of the portal vein (A). Cavernous transformation of the PV (arrow) with strong blood flow (B). Cavernous transformation (arrow) after a complete portal vein

which are gas microbubbles (e.g., sulfur hexafluoride; octafluoropropane; Perfluorobutane) surrounded by a lipid or albumin shell providing stability to the com- thrombosis depicted with axial ceMRI (C) and coronal ceCT (D). Early cavernous transformation of the PV about 4 weeks after an acute episode of pain with formation of collateral vessels (arrow) (E). Chronic portal vein thrombosis where only a fibrous cord (arrow) instead of the portal vein (F).

pound [23]. The microbubbles are similar to red blood cells in size and remain exclusively in the vascular system (purely intravascular contrast agents). Upon insonation 


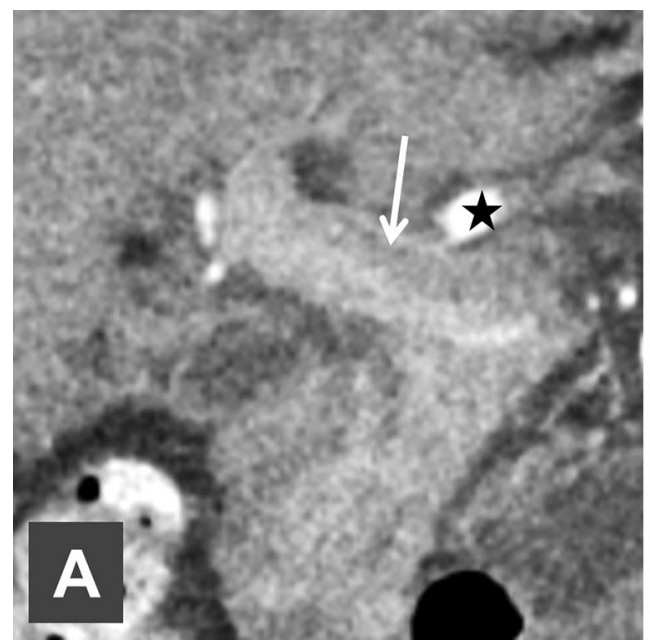

Fig. 4. A 65-year-old patient undergoing a CT scan of the abdomen due to an unclear lesion in the liver. In the portal venous phase $60 \mathrm{~s}$ post contrast injection there is a hypodense band close to the upper contour of the main trunk of the portal vein (arrow, A). However, in the late phase of the examination $180 \mathrm{~s}$ post contrast injection this is not seen any more (arrow, B). Obviously, the hypodensity close to vessel

the microbubbles oscillate creating harmonics (non-linear behavior) which enhance echo signals that can be detected by specific software, permitting perfusion visualization at real time [23]. Given its improved sensitivity of CEUS versus standard US in vascular evaluation, CEUS has gained ground in diagnosing PVT in complex cases (e.g., patients with decompensated cirrhosis who can have a very slow flow in the portal vein that can be erroneously confounded with PVT; patients with recent liver transplantation) [24]. Furthermore, CEUS markedly improved the characterization of PVT, and allows differentiating thrombosis from malignant invasion of the PV in patients with hepatocellular carcinoma (HCC) $[25,26]$ (see "Differentiating thrombosis from tumorous invasion of the portal vein").

\section{Contrast-enhanced computed tomography}

Conventional multislice, ceCT is the second most common imaging test to identify PVT after US and may be the best method to define the anatomy of the vascular changes. Multiphasic CT has four phases: pre-contrast, arterial, portal and late. Attaining images at the adequate time (portal phase) is mandatory to avoid pitfalls. Images acquired during the late arterial phase are not ideal for the diagnosis of PVT as this phase is too early for a full contrast filling of the portal vein. In this phase, contrast inflow phenomenon may lead to false-positive results as well. Moreover, in case of reduced or slow portal vein inflow (often observed in decompensated cirrhosis), a delayed contrast arrival to the PV can lead to a false

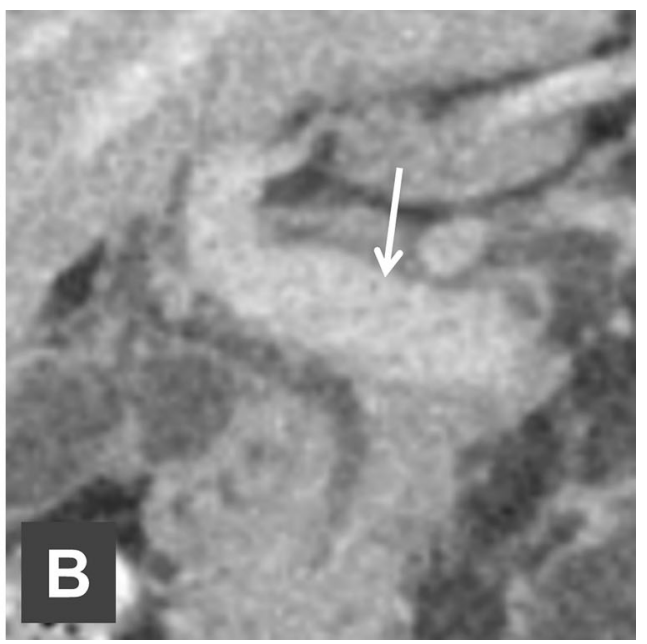

wall was due to an inflow phenomenon of the contrast medium as the portal venous phase was acquired too early with a still high contrast in the liver artery (black star in A). If available, late contrast phases should be compared with portal venous phases before the diagnosis of a portal venous thrombosis to avoid false-positive results.

appearance of a filling defect on CT (Fig. 4). To avoid pitfalls during imaging, two situations must be distinguished. If only one contrast phase is being acquired, it is recommended to acquire the portal phase about 10-15 s later than the usual 60-70 s after contrast injection [18]. In the case that several contrast phases are acquired, as it is usually the case for protocols for the imaging of the liver and differentiation of focal liver lesions, a venous phase should be obtained approximately $90 \mathrm{~s}$ after contrast injection. Comparing both the portal venous and venous phase usually allows a differentiation between an inflow phenomenon and a real thrombotic material within the portal vein [18].

Typically, in non-contrast-enhanced CT, PVT is seen as isodense to adjacent soft tissue. A hyperdense aspect can be shown if thrombosis occurred within a month. This elevated CT attenuation value has been attributed to the protein fraction of hemoglobin in the clot [27]. Following intravenous administration of iodinated contrast on CT, a thrombus appears as a low density, nonenhancing defect within the PV lumen [27]. Moreover, dynamic CT shows a filling defect partially or totally occluding the (Figs. 1,2) vessel with rim enhancement of the wall [27]. Additionally, ceCT can show peripheral bands of parenchyma with higher density in the arterial phase fading into isodensity in the portal phase, defined as transient hepatic arterial difference (THAD). This typically spares the juxta-hilar area where blood supply from the cavernous transformation of the PV to the liver tissue is sufficient [27]. Other signs include decreased venous enhancement in the liver during the portal phase 
and enlargement of the thrombosed PV [27]. As for other imaging modalities, indirect markers of PVT are the presence of cavernous transformation of the PV or fibrous cord, porto-systemic collateral vessels and arterioportal shunts [28] (Fig. 3).

Non-cirrhotic patients with cavernous transformation of the PV can present hepatic morphological changes described as "atrophy-hypertrophy complex of the liver" with a larger central part of the liver (segment IV and caudate lobe). It can be speculated that the supply of portal venous blood flow from the cavernous transformation is not sufficient in the subcapsular or peripheral areas, leading to their atrophy and inducing compensatory hypertrophy of the remaining segments [29].

$\mathrm{CeCT}$ has added value to map the mesenteric vasculature and to identify secondary bowel complications, such as bowel ischemia, and to identify contributing diseases like a malignant mass, loco-regional infection/ inflammation, or signs of cirrhosis.

The principal downfalls of ceCT imaging are the necessity of administering considerable volumes of potentially nephrotoxic iodinated contrast agents and exposure to ionizing radiation. In this regard, the incidence of contrast-induced nephropathy (CIN) is estimated around 5\% [30, 31]). In cases of pre-existing renal failure or iodine intolerance, shifting to MR imaging is reasonable. Given the concerns regarding high radiation exposure, omitting the unenhanced scans could be an effective strategy. This can be achieved by dual-energy CT (DECT) with iodine quantification in tissues, which offers a direct evaluation of vascularization, independent of attenuation [32].

\section{Magnetic resonance imaging}

CeMR is a sensitive and specific technique (accuracy close to 95\%) [33] to detect partial PVT and occlusion of the main portal venous vessels, and to map the portosplenic collaterals. Unenhanced T1- and T2- weighted sequences are not ideal for the diagnosis of PVT, suggesting that the thrombus signal intensity varies based on the sequences used, theoretically resulting from the age of the thrombus or the low intrinsic contrast of these images compared with post contrast images. Flow artifacts have also been shown to be problematic on noncontrast images.

Therefore, it is consensual that the use of contrast images for the diagnosis of PVT is essential. The clot appears isointense to hyperintense on unenhanced T1weighted images, and usually has a more intense signal on T2w images, while older clots appear hyperintense only on T2-weighted images (Fig. 2). Also, gradient-echo MR might help to better evaluate any equivocal findings on spin-echo MR image [34]. The main difference between non-thrombosed diminutive PV resulting from cirrhosis and recanalized chronic thrombosis is the in- creased wall thickness of the PV in the setting of recanalization [33].

As for the safety of gadolinium-based contrast agents (GBCAs), there had been many concerns about the onset of nephrogenic systemic fibrosis (NSF) as a result of the release of gadolinium from the complex structure [35]. Recent research largely restored the confidence in the safety of GBCAs has been widely restored. It has been shown that the development of NSF can be largely avoided by avoiding GBCAs with a linear an less stable structure in patients with chronic kidney disease stages 4 and 5, or using more stable macrocyclic GBCAs $[36,37]$.

MRI-true fast imaging with steady-state precession (true FISP) might overcome the need for a contrast agent administration, the difficulty of contrast injection in cases of poor venous access and the degradation of the images by respiratory motion. To surpass these limitations, recent studies have demonstrated the efficacy of non-ceMR portography in the diagnosis of PVT. NonceMR angiography can depict the PV with the combined usage of a respiratory-triggered three-dimensional (3D) balanced steady-state free precession (bSSFP) sequence and a time spatial labeling inversion pulse (T-SLIP) [38]. The application of Half-Fourier fast spin-echo scanning with T-SLIP enabled selective visualization of the PV without an exogenous contrast agent in healthy individuals [38]. This provides a high signal-to-noise ratio (SNR) per unit time and can produce an image with high resolution. Balanced SSFP allows visualization of the vessels without the use of an intravenous contrast agent in a short acquisition time [39]. Recently, a study showed that the flow-out method produced high-quality images of the intrahepatic PV and peripheral branches during free breathing without an exogenous contrast agent [40].

Diffusion-weighted MR imaging (DW-MRI) that can also be applied in the liver is based on the Brownian motion of water within different tissues [41]. Tumor tissue usually contains a high cellularity with densely packed tumor cells with a decrease in extracellular spaces that lead to a restricted diffusion [42]. DW-MRI consists of an acquisition of at least two $b$-values (in the liver usually three values: 50,300 and $800 \mathrm{~s} / \mathrm{mm}^{2}$ ) which allows the calculation of apparent diffusion coefficient (ADC) maps. In tissues with tightly packed cells like malignancies, Brownian motion is less than in an environment with a lower degree of compartmentalization and therefore diffusion is impeded, appearing bright on DW-MR images and darker in the ADC map [43]. The extent of diffusion impediment can be analyzed qualitatively on the ADC map and can also be measured objectively.

Magnetic resonance cholangiopancreatography (MRCP) is the technique of choice to be applied upon suspicion of bile ducts abnormalities which can occur as a result of long-lasting cavernous transformation of the 

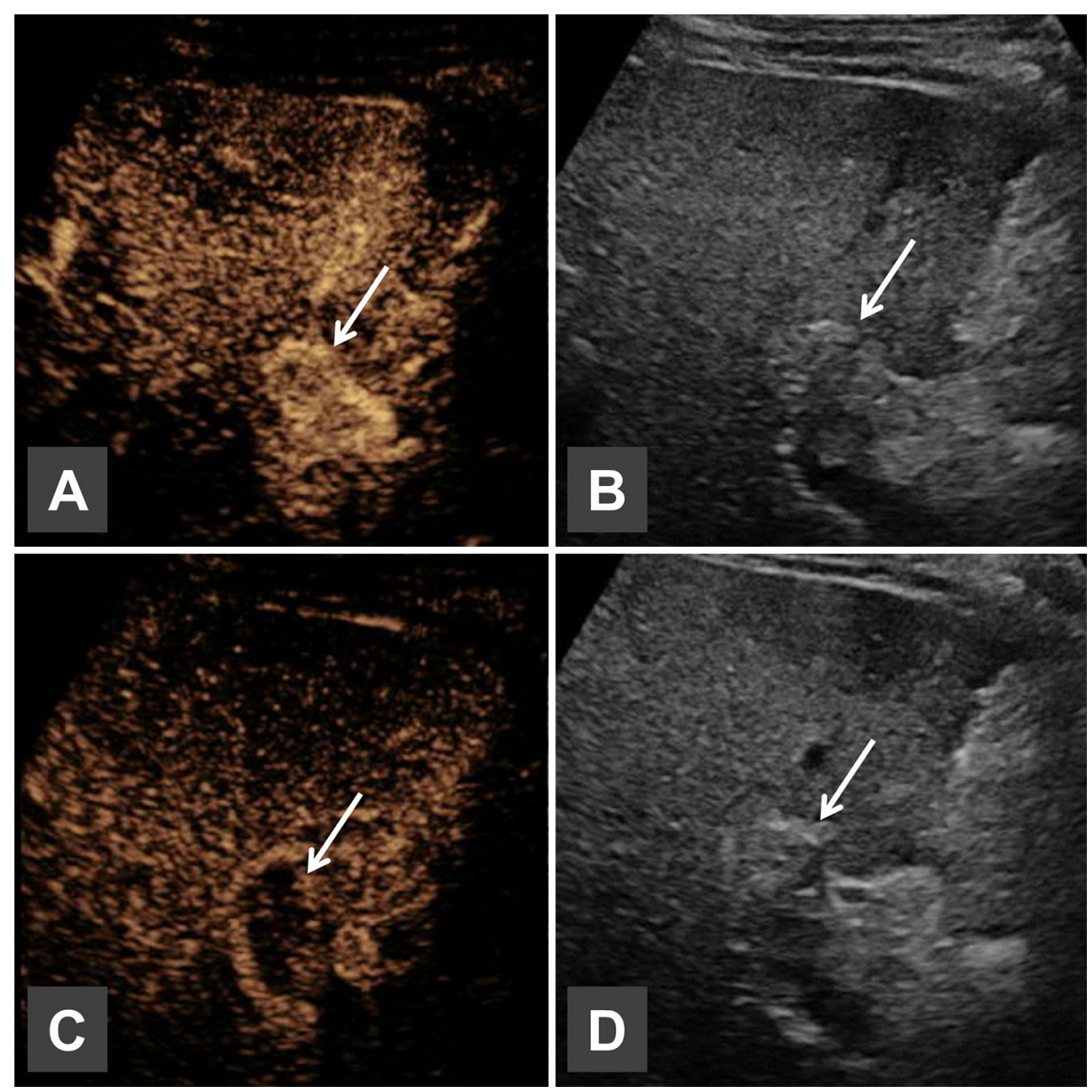

Fig. 5. Contrast-enhanced ultrasound revealing a tumor invasion of the main trunk of the portal vein with an initial "wash-in" of contrast medium (arrows in A and B) followed by a "wash-out" afterwards (arrows in C and D).

PV (see "Mapping collaterals and identifying and grading portal cholangiopathy" and Table 2).

\section{Indirect intraarterial portography and direct transparietal access}

The invasive angiographic approach is reserved for rare cases with equivocal results on noninvasive imaging. In the management of symptomatic splanchnic venous thrombosis, it is used in conjunction with endovascular therapeutic procedures (see below).

\section{Specific questions with clinical implications to be addressed by imaging on diagnosis and in the follow-up}

\section{Differentiating thrombosis from tumorous invasion of the portal vein}

Tumorous invasion of the portal vein is commonly associated with hepatocellular carcinoma, particularly of the infiltrative type, and can occur in other intrahepatic and extrahepatic cancers, which should be always investigated and ruled-out in patients with newly diagnosed PVT. The diagnosis of malignant PV invasion has very relevant prognostic and therapeutic implications (e.g., exclusion from LT program in patients with HCC) and attention should be paid to signs that can help differentiating between thrombosis and tumorous invasion in patients with known HCC. Signs suggesting tumourous invasion on any imaging method include direct visualization of a tumoural mass adjacent to the PV, disruption of the walls of the vessel and diameter exceeding $23 \mathrm{~mm}$ [25]. Hyperenhancement in the arterial phase and washout in the delayed phase are typical features of HCC and HCC-related PV invasion on CEUS (Fig. 5), ceCT, and ceMR. CDUS and spectral Doppler can sometimes directly visualize arterial vessels within the material filling the portal vein (Fig. 6), but have a limited accuracy (sensitivity 82.5\%; specificity 100\%). CEUS has accuracy close to $100 \%[25,26]$. CeCT might show the typical "thread and streak pattern" [28], but 

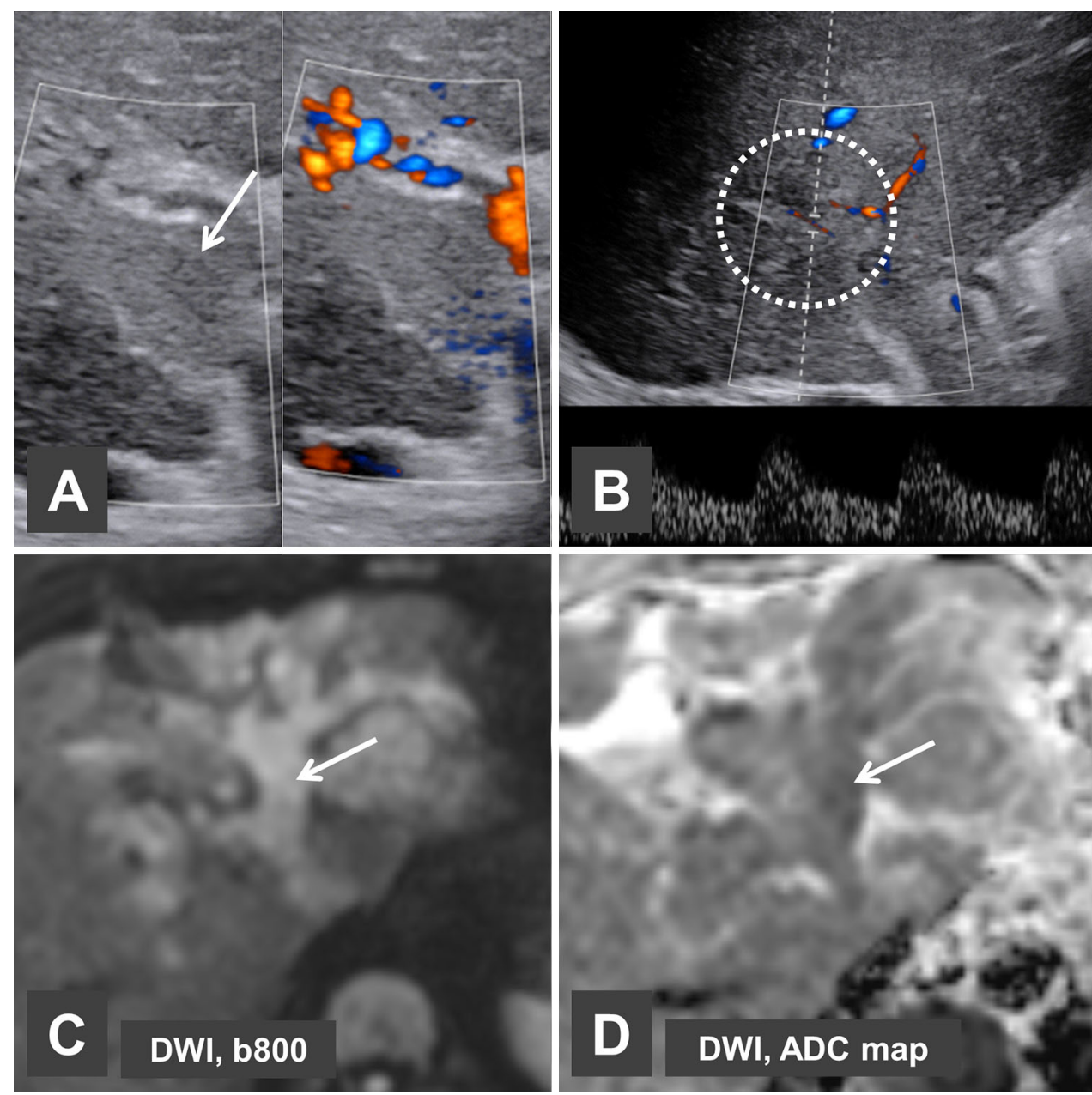

Fig. 6. A 55-year-old patient with a known hepatocellular carcinoma (HCC) that is diffusely infiltrating the liver parenchyma in the liver segments II, III, and IVa/b. An ultrasonography shows a widening of the portal vein that is filled with hyperechogenic tumor mass $(\mathbf{A})$. Doppler ultrasonography reveals a vascularization of the tumor mass with arterial

due to its limited time-resolution it might miss the arterial hyperenhancement. The quantification of thrombus iodine density in the portal venous phase by dual-energy multi-detector CT with iodine quantification appears as a promising new imaging technique for distinguishing between thrombosis and neoplastic portal vein thrombus material [44, 45].

Regarding MRI for differentiating thrombosis vs. neoplastic invasion, T2-weighted imaging is a powerful tool as tumor thrombi frequently have a heterogeneous signal and a hyperintense appearance in T2- weighted images. A subsequent confirmation can be obtained by an uptake of contrast medium in dynamic fat saturated T1-weighted imaging. A pilot study suggested that DWMRI can differentiate thrombosis from neoplastic invasion material [46]. In this study, neoplastic thrombosis showed low ADC values in the ADC map which were vessels (circle, B). Diffusion-weighted MRI (DW-MRI) has a high signal in the b800 image within the tumor tissue (arrow, C). The corresponding ADC map shows a low signal indicating a high diffusion restriction due to tightly packed tumor cells (arrow, D).

similar to those observed in HCC (Fig. 6). On the contrary, thrombosis showed high ADC values [46]. However, other studies revealed that signal-intensity characteristics on DWI and measured ADC values do not differentiate benign from malignant PVT reliably [47, 48].

Fine needle biopsy of the thrombus either percutaneously [49] or during endosonography [50] can be safely performed in doubtful cases to achieve a final diagnosis.

\section{Diagnosing/excluding cirrhosis}

Patients with liver cirrhosis are prone to develop PVT, and a reduced PV flow velocity (i.e., $<15 \mathrm{~cm} / \mathrm{sec}$ ) is an independent predictor of the development of PVT [13] in this population. In patients with newly diagnosed PVT, the diagnosis of cirrhosis should be always taken into 


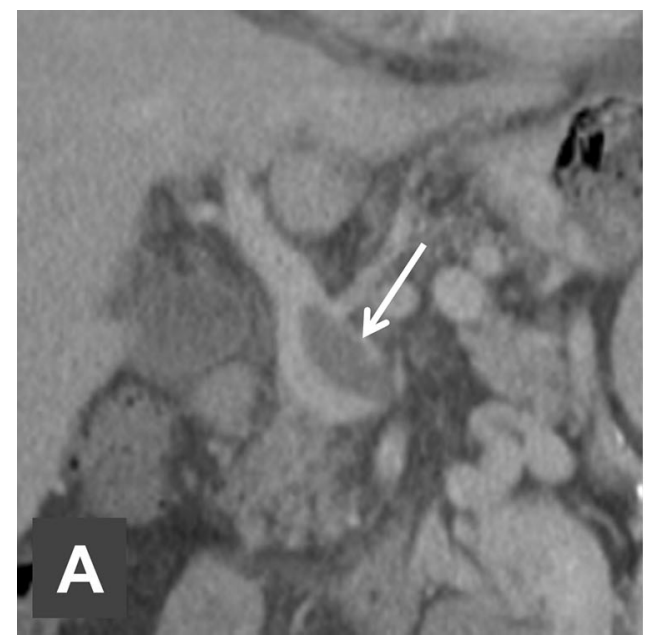

Fig. 7. A 45-year-old patient with an acute partial thrombosis of the portal vein close to the confluens in a former CT scan from the year 2010 (arrow, A). A follow-up CT

consideration. Imaging signs of cirrhosis include liver surface nodularity on ultrasound [51] or CT [52], as well as morphological changes (caudate lobe hypertrophy; atrophy of the right liver lobe; curling of hepatic veins). However, similar changes can be observed in patients with long-lasting chronic PVT (Fig. 1), and should be considered not $100 \%$ specific in this setting. Liver stiffness measurement by ultrasound elastography provided a new effective tool to support the diagnosis of cirrhosis vs. non-cirrhosis with over $90 \%$ accuracy [53]. Liver stiffness is typically increased in patients with cirrhosis and normal or almost normal in patients with PVT [54].

\section{Discriminating between recent/acute and chronic PVT}

Distinguishing between a recent and chronic setting is pertinent to decision-making regarding the optimal therapy, which is aimed at achieving recanalization and avoid thrombus extension. Recent/acute PVT shows a higher rate of recanalization after anticoagulation in comparison to chronic thrombosis [7, 55]; furthermore, persistence or absence of a portal remnant is crucial to plan an eventual transjugular intrahepatic porto-systemic shunt (TIPS) and to attempt PV recanalization.

The natural progression of PVT is a dynamic process that extends from recent thrombus to cavernous transformation of PV with further progression to the intraand extrahepatic porto-mesenteric venous system. This course can occur in a few days from partial to complete obstruction, to fibrotic cord, finalizing in 3-5 weeks. Cavernous transformation of the PV development could occur between 6 and 20 days after an acute onset and once cavernous transformation of the PV is detected, patients are regarded as having entered the chronic stage

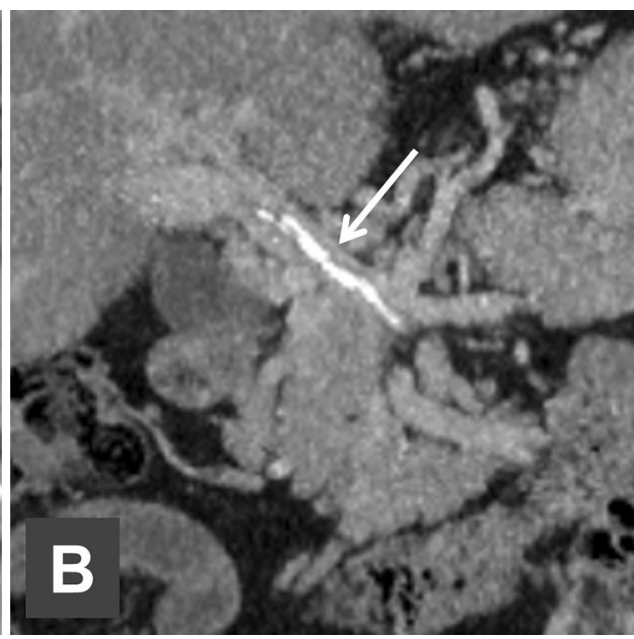

scan in 2016 shows an extensive calcification of the thrombotic material along the course of the portal vein (arrow, B).

[5]. Complications, namely, signs of $\mathrm{PH}$ and portal cholangiopathy (PC) are detected subsequently, in later stages. The presence of calcifications within the wall of the vessel or the thrombus is a pathognomonic finding that can only be seen using US or CT (Fig. 7), but not MRI. Furthermore, in non-cirrhotic patients without underlying hematological disorders, an acute onset can be supported by the absence of porto-systemic collaterals and a normal or mildly increased spleen size. There are certain situations where it is unfeasible to determine the age of the thrombus, such as when a hypoechoic complete or partial thrombus is detected in a vessel without collaterals and with a normal size.

\section{Mapping collaterals and identifying and grading portal cholangiopathy}

$\mathrm{CeCT}$ is the technique of choice to map the porto-systemic and porto-portal collateral vessels. Post-processing tools such as maximum intensity projection, volume rendering and surface shaded display are useful adjuncts to map the system of porto-portal and porto-systemic collaterals, and study their relationship with the bile ducts [56]. A direct compression of the cavernous transformation of the PV on the intra- and/or extrahepatic biliary ducts can lead to obstruction and dilatation, and gallbladder abnormalities which is termed "portal cholangiopathy" (PC) or "portal biliopathy" [56, 57]. It is diagnosed in imaging in over $80 \%$ of patients with EHPVO, but clinical consequences such as cholecystitis, cholangitis and jaundice are uncommon [57]. Abnormalities of the biliary tree can be classified into different degrees of severity: no abnormalities; grade I (irregularities or angulations of the biliary tree); grade II (indentations or strictures without dilation); and grade 
Table 3. Most commonly reported indications, advantages, and disadvantages of the main endovascular treatment modalities used in patients with acute or chronic PVT

\begin{tabular}{|c|c|c|c|}
\hline & Indications & Advantages & Disadvantages \\
\hline $\begin{array}{l}\text { Thrombolysis } \\
\text { Direct }^{\text {a }} \text { (transhepatic or transsplenic) } \\
\text { Indirect (through the superior mesenteric artery) }\end{array}$ & $\begin{array}{l}\text { Large recent PVT } \\
\text { without PH, } \\
\text { coagulopathy, cir- } \\
\text { rhosis } \\
\text { Unsuccessful medi- } \\
\text { cal therapy } \\
\text { - Unsuccessful med- } \\
\text { ical therapy } \\
\text { - Failed or not } \\
\text { impossible direct } \\
\text { approach } \\
\text { - Extensive mesen- } \\
\text { teric thrombosis }\end{array}$ & $\begin{array}{l}\text { - Relatively easy and fast } \\
\text { - Treat small tributary } \\
\text { mesenteric thrombosis } \\
\text { - Can be used as adjunct to } \\
\text { the direct technique }\end{array}$ & $\begin{array}{l}\text { - High risk of bleeding from the } \\
\text { access site } \\
\text { - Less dose delivery vs. indirect } \\
\text { - Allows to combine } \\
\text { thrombectomy } \\
\text { angioplasty/stent } \\
\text { - Higher dose of thrombolytic } \\
\text { agent required, and conse- } \\
\text { quently increased systemic } \\
\text { toxicity/risk of bleeding vs. } \\
\text { direct technique } \\
\text { - Increased infusion time } \\
\text { - Does not allow to combine } \\
\text { thrombectomy/ angioplasty/ } \\
\text { stenting with the same access }\end{array}$ \\
\hline Thrombectomy & $\begin{array}{l}\text { - Used as a self-s- } \\
\text { tand technique in } \\
\text { patients with con- } \\
\text { traindications for } \\
\text { thrombolysis } \\
\text { - Used in combina- } \\
\text { tion with throm- } \\
\text { bolysis in selected } \\
\text { cases }\end{array}$ & $\begin{array}{l}\text { - Safe in patients with high } \\
\text { risk of bleeding } \\
\text { - If combined to thrombol- } \\
\text { ysis: shorter thrombolysis } \\
\text { dose and time }\end{array}$ & $\begin{array}{l}\text { - Increased lesion of vessel and } \\
\text { re-thrombosis } \\
\text { - Risk of pulmonary embolism } \\
\text { and bradycardia }\end{array}$ \\
\hline $\begin{array}{l}\text { Transjugular transhepatic approach } \\
\text { and TIPS placement }\end{array}$ & $\begin{array}{l}\text { - Symptomatic re- } \\
\text { cent PVT, without } \\
\text { bowel ischemia } \\
\text { - Recent PVT with } \\
\text { cirrhosis and PH: } \\
\text { ascites } \pm \text { coagu- } \\
\text { lopathy } \\
\text { - Can be attempted } \\
\text { in chronic PVT if } \\
\text { a remnant of the } \\
\text { PV or a dominant } \\
\text { large intrahepatic } \\
\text { portal collateral is } \\
\text { present and pro- } \\
\text { vided a landing } \\
\text { zone (patent sple- } \\
\text { nic and/or SM } \\
\text { vein) exists } \\
\text { - Previous TIPS with } \\
\text { re-thrombosis or } \\
\text { extension to SMV }\end{array}$ & $\begin{array}{l}\text { - Lower bleeding risk vs. } \\
\text { direct transparietal and } \\
\text { transsplenic approach } \\
\text { - Allows to combine } \\
\text { thrombolysis and } \\
\text { thrombectomy } \\
\text { - TIPS placement: allows } \\
\text { for faster recanalization } \\
\text { of thrombosis (fast flow) } \\
\text { and resolves PH }\end{array}$ & $\begin{array}{l}\text { - Usually long procedure } \\
\text { - Risk of arrhythmias (catheter } \\
\text { pass in the right atrium) } \\
\text { - Need for ultrasound guidance } \\
\text { to the thrombosed portal } \\
\text { vein } \\
\text { - Risk of liver capsule puncture } \\
\text { (intraperitoneal bleeding) } \\
\text { and hemobilia low but not } \\
\text { inexistent } \\
\text { - Risk of intrahepatic hemato- } \\
\text { ma } \\
\text { - In chronic PVT: technically } \\
\text { challenging procedure, } \\
\text { requiring specific expertise }\end{array}$ \\
\hline
\end{tabular}

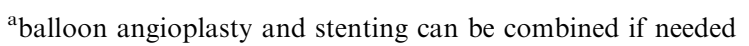

III (strictures with dilation; biliary dilation if intrahepatic ducts $>4 \mathrm{~mm}$ or extrahepatic duct $>7 \mathrm{~mm}$ ) [57]. Severe PC developed in $30 \%$ of patients with acute PVT within 1 year [57]. Major differential diagnoses of PC include hilar cholangiocarcinoma and primary sclerosing cholangitis, which should always be carefully excluded [56]. Whilst US and CT can visualize PC, MRCP remains the modality of choice for its complete characterization. MRCP should be performed at diagnosis in patients with chronic PVT and after 9-12 months of acute PVT if recanalization is not achieved with AC. If grade III portal cholangiopathy has not developed at the 12 month of follow-up, no further examination is needed, as progression is unexpected [57]. Endoscopic ret- rograde cholangiopancreatography is reserved only for those requiring therapeutic intervention. Endoscopic ultrasonography (EUS) is not routinely employed and is generally recommended only when differentiation between fibrous collateral tuft, stones, and tumors is impossible with other modalities [58].

\section{Role of imaging in treatment}

\section{Medical therapy}

Anticoagulation (AC) with vitamin $\mathrm{K}$ antagonists or with heparins is the current mainstay of treatment of PVT. Besides achieving recanalization in $39 \%-80 \%$ of 


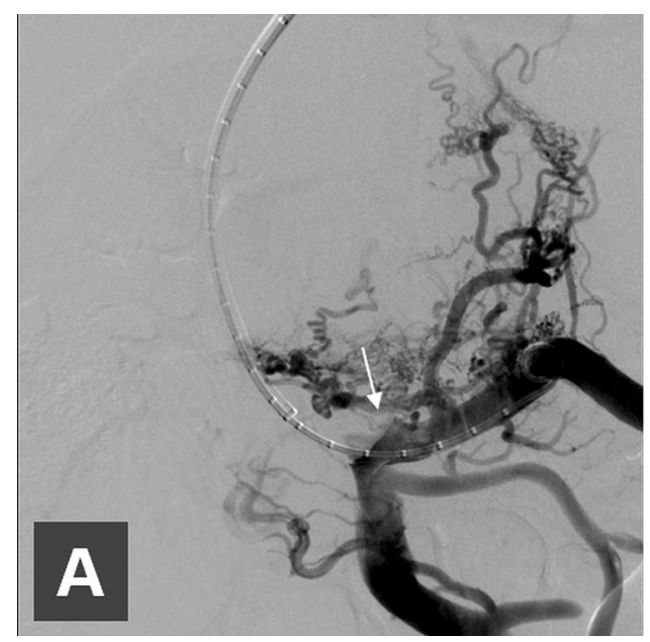

Fig. 8. Isolated acute intra- and extrahepatic portal vein thrombosis in a patient with liver cirrhosis candidate to liver transplantation. A Direct portography, using the transjugular intrahepatic porto-systemic shunt (TIPS) access with complete thrombotic occlusion of the portal vein down to the confluens (arrow), porto-systemic pressure gradient (PPG)

cases, it avoids progression, re-thrombosis, and secondary complications $[5,17]$.

The use of direct oral anticoagulant drugs (DOACs, such as, rivaroxoban, dabigratan, apixaban) has been growing in the context of PVT, revealing efficacy, and safety $[59,60]$. However, DOACs remain off-label in patients with PVT and their use in patients with decompensated cirrhosis is contraindicated due to insufficient data. Permanent AC should be offered in cases of prothrombotic conditions, or in PVT extending to the SMV, as the benefits clearly outweigh the risks [17]. Continued AC in transplant candidates is recommended. In patients not responding to $\mathrm{AC}$ or when the SMV is affected, alternative therapeutic options should be considered [17].

Recanalization on AC is not universal and usually does not occur beyond the first 6 months of therapy. Furthermore, bleeding risks are not negligible. Therefore, an accurate follow-up on imaging is needed to guide clinical decisions whether to keep or suspend AC.

\section{Minimally invasive vascular techniques}

Table 3 clarifies the main reported indications, advantages and disadvantages of the principal endovascular treatment modalities. Imaging plays a crucial role on taking the decision of attempting endovascular treatment. A remnant of intrahepatic portal vein is required to attempt a transhepatic approach, and in the authors' experience the use of ultrasound improves the selection of patients in whom portal vein access can be successfully achieved. On the other hand, a complete and extensive

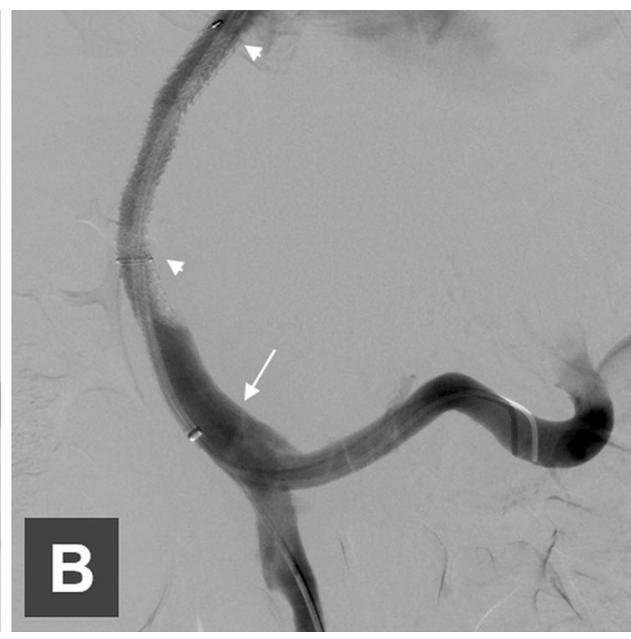

$22 \mathrm{mmHg}$. After thrombus fragmentation using a Dormia (C) basket, thrombus aspiration and intrahepatic Viatorr@ stent insertion (TIPS) the common portal vein as landing zone for transplantation was free of thrombus. B Completion portography with brisk contrast flow via common portal vein (arrow) and TIPS (arrow head), PPG $4 \mathrm{mmHg}$.

cavernomatous transformation of $\mathrm{PV}, \mathrm{SV}$, and SMV prevents an endovascular treatment.

Direct access to the portal vein can be achieved by different approaches, namely (a) percutaneous transhepatic puncture; (b) percutaneous transplenic puncture; (c) transjugular intrahepatic puncture. Transileocolic, and omental vein approaches are rarely performed [61]. The access to the portal vein can take advantage of ultrasound guidance. Percutaneous transsplenic approach is reserved for patients for whom conventional transhepatic or transjugular intrahepatic approaches are difficult or unfeasible, as it carries a higher risk of bleeding.

In contrast to the transjugular, transhepatic access in a non-thrombosed PV, sonographic guidance is needed for safe access a thrombosed PV system.

No head-to-head studies comparing endovascular techniques with anticoagulation and recanalization with vs. without thrombolysis are currently available.

\section{Thrombolysis}

Catheter-directed thrombolytic (CDT) therapy with or without thrombectomy has been proposed for patients with thrombus extension or worsening symptoms while on therapeutic AC, and in patients with impending or ongoing bowel ischemia due to thrombosis, in cases with an acceptably low risk of bleeding. Local clot dissolution with low-dose thrombolytic therapy (e.g., $1 \mathrm{mg} / \mathrm{h} \mathrm{rt}-\mathrm{PA}$ for a max of $48 \mathrm{~h}$ ) is effective and safe with low risk of bleeding [62]. Infusion involves placement of a 4 or $5 \mathrm{Fr}$ infusion catheter with multiple side holes across the thrombus. A thrombolytic agent is administered through the catheter 
and heparin is administered through the sheath [61]. Direct percutaneous transhepatic or transsplenic portal venous access without TIPS is uncommon as it lacks restoration of brisk venous outflow. The method of choice is the transjugular, transhepatic access to reach the porto-mesenteric thrombus, followed by transhepatic stent placement (TIPS) to enhance venous outflow. This method is more efficient, faster, and requires lower doses of thrombolytic agent, in comparison with the indirect approach in which thrombolytic agents are administered into the superior mesenteric artery through the femoral or radial artery. The combination of mechanical thrombectomy with CDT (PMCT) has the advantage of lysing smaller thrombi not targeted by mechanical thrombectomy. Thromboses of both portal and mesenteric veins are typically associated with more congestion and fewer collateral vessels, suggest that loco-regional CDT are favorable in these circumstances. Main reason of CDT treatment failure is the low likelihood of dissolution in cases of significant thrombus organization. The choice of thrombolysis agent should be considered with local urokinase ${ }^{\circledR}$ or alteplase ${ }^{\circledR}$ (rt-PA) in combination with unfractionated heparin. Post-procedure continuous oral AC therapy and close imaging surveillance are indicated.

\section{Thrombus fragmentation and thrombectomy}

Thrombus fragmentation to destruct the clot (for more efficient thrombolysis) can be achieved mechanically, with pigtail catheter, balloons, or special devices such as Dormia baskets or suction techniques [63]. Aspiration of the fragmented thrombus can be performed using 8 Fr aspiration catheter or dedicated large sized thrombectomy devices. This method is primarily indicated in patients with high hemorrhagic risk or relative contraindications to thrombolytic agents. When used in conjunction with CDT (pharmacomechanical thrombolysis, PMCT), it decreases the required dose of alteplase ${ }^{\circledR}$ and the need for prolonged infusion [64]. Nonetheless, there is a risk of vessel injury and re-thrombosis [65]. Pulmonary embolism and bradychardia related to PMCT have been described if performed after stenting of the transhepatic access (TIPS) to increase venous outflow.

\section{Angioplasty and stenting}

Balloon angioplasty and stenting are meaningful in case of residual/refractory thrombosis and stenosis of the porto-mesenteric venous system, with or without previous thrombolysis/thrombectomy. In cases of tumorous invasion of the PV, palliative stents, particularly selfexpanding, can be effective.

\section{Transjugular intrahepatic porto-systemic shunt (TIPS)}

The transjugular, transhepatic porto-systemic (TIPS) (Fig. 8) access has a clear advantage over direct tran- shepatic or transsplenic access in patients with ascites, splenomegaly, or coagulopathy. It is, therefore, preferred in patients with PVT, cirrhosis and PH. The bleeding risk is lower as compared to direct transhepatic or transsplenic access and it provides access for embolization of the esophageal and gastric varices. After TIPS, PH is no longer present and flow velocity through the portal system is increased, reducing recurrence of PVT [66]. In patients with extensive PVT and bowel ischemia, endovascular CDT is usually combined with a TIPS procedure to increase venous outflow.

In patients with chronically occluded PVT, TIPS placement is challenging. Transsplenic PV recanalization followed by TIPS is a potentially safe and effective method to treat chronic PVT and improve transplant candidacy [67]. This route might be considered in patients in whom standard approaches are unsuccessful, but it carries a higher risk of bleeding.

In complex cases, a superior mesenteric arteriography may assist in mapping the anatomy and delineating a treatment plan.

AC therapy is normally maintained after TIPS in patients with PVT and cirrhosis. In the only randomized controlled trial available comparing TIPS + AC vs. TIPS only in this population (either acute or chronic), both strategies showed a high 12-month patency rate (83.9\% in the combination vs. $71.8 \%$ in TIPS only, $p=0.25)[68]$.

\section{Role of imaging in patient follow-up}

Imaging is a crucial tool in monitoring the complications of portal hypertension and the response to AC. Whenever a major episode of decompensation (e.g., variceal bleeding), ceCT, or ceMR should be performed to evaluate possible re- thrombosis episodes, plot the patent vessels, and porto-systemic collaterals (commonly ectopic), and, ultimately, to assess whether endovascular procedures (e.g., TIPS; embolization of collaterals) or shunt surgery are technically feasible [17]. On follow-up, images should be always compared to the previous ones, and both recanalization and re-thrombosis/new thrombosis should be clearly reported to facilitate clinical decisions.

Since recanalization of the PV occurs up to 6 months after AC, a follow-up imaging study after 6 months of $\mathrm{AC}$ is recommended [17]. The first imaging technique used upon starting AC should be used [17]. After this first re-assessment, there is no consensus on which technique and interval should be used, and follow-up should be individualized according to the patient's risk factors, the institution's experience and risks of repeated use of radiation and iodinate contrast $[17,18]$. However, it should be considered that recanalization of splenic vein and mesenteric veins can take longer than 6 months, Therefore, in patients presenting with SV and/or SMV at 
our institution we consider useful a further assessment at 12 month either by ceCT or ceMRI [7]. It is important therefore to remark that whenever technically feasible (good ultrasound visualization) CDUS should be used to follow-up patients with PVT.

\section{Conclusions}

As described in this review, imaging has a fundamental role in the diagnosis and management of patients with PVT. Imaging contributes to promptly identify and characterize thrombosis, detecting the underlying disease/conditions and improving prognostic stratification, all crucial aspects that should be taken into consideration on attempting recanalization. Endovascular techniques offer the ability to restore PV patency, and should be considered in acute/recent PVT when thrombosis is refractory to $\mathrm{AC}$ and when complications of $\mathrm{PH}$ have already developed. After treatment, imaging follow-up is the key to identify patients progressing despite first-line therapy with $\mathrm{AC}$, and to monitor maintenance of patency in patients in whom AC is stopped. Lack of highquality evidence is a major problem to guide therapy in this field. Future well-designed prospective studies should provide evidence to define the best therapeutic algorithm for acute and chronic PVT. Due to the complexity and severity of this condition, a multidisciplinary approach with hepatologists, diagnostic radiologists, interventionalists and surgeons is critical to elaborate a standard diagnostic and therapeutic protocol and to ensure successful outcomes.

\section{Compliance with ethical standards}

Funding This article was funded by the University of Berne (Grant UniBe-ID-2015).

Conflict of interest Susana G. Rodrigues declares that she has no conflict of interest; Martin H Maurer declares that he has no conflict of interest; Iris Baumgartner declares that she has no conflict of interest; Andrea De Gottardi declares that he has no conflict of interest; Annalisa Berzigotti declares that she has no conflict of interest.

Ethical approval This article does not contain any studies with human participants performed by any of the authors.

\section{References}

1. Ogren M, Bergqvist D, Bjorck M, et al. (2006) Portal vein thrombosis: prevalence, patient characteristics and lifetime risk: a population study based on 23,796 consecutive autopsies. World $\mathbf{J}$ Gastroenterol 12:2115-2119

2. Condat B, Valla D (2006) Nonmalignant portal vein thrombosis in adults. Nat Clin Pract Gastroenterol Hepatol 3:505-515

3. Webster GJ, Burroughs AK, Riordan SM (2005) Review article: portal vein thrombosis - new insights into aetiology and management. Aliment Pharmacol Ther 21:1-9

4. Denninger MH, Chait Y, Casadevall N, et al. (2000) Cause of portal or hepatic venous thrombosis in adults: the role of multiple concurrent factors. Hepatology 31:587-591

5. de Franchis R, Baveno VIF (2015) Expanding consensus in portal hypertension: report of the Baveno VI Consensus workshop: stratifying risk and individualizing care for portal hypertension. J Hepatol 63:743-752
6. Primignani M, Martinelli I, Bucciarelli P, et al. (2005) Risk factors for thrombophilia in extrahepatic portal vein obstruction. Hepatology 41:603-608

7. Plessier A, Darwish-Murad S, Hernandez-Guerra M, et al. (2010) Acute portal vein thrombosis unrelated to cirrhosis: a prospective multicenter follow-up study. Hepatology 51:210-218

8. Bureau C, Laurent J, Robic MA, et al. (2016) Central obesity is associated with non-cirrhotic portal vein thrombosis. J Hepatol $64: 427-432$

9. Wanless IR, Wong F, Blendis LM, et al. (1995) Hepatic and portal vein thrombosis in cirrhosis: possible role in development of parenchymal extinction and portal hypertension. Hepatology 21:1238-1247

10. Tripodi A (2015) Hemostasis abnormalities in cirrhosis. Curr Opin Hematol 22:406-412

11. Tsochatzis EA, Senzolo M, Germani G, Gatt A, Burroughs AK (2010) Systematic review: portal vein thrombosis in cirrhosis. Aliment Pharmacol Ther 31:366-374

12. Nery F, Chevret S, Condat B, et al. (2015) Causes and consequences of portal vein thrombosis in 1,243 patients with cirrhosis: results of a longitudinal study. Hepatology 61:660-667

13. Zocco MA, Di Stasio E, De Cristofaro R, et al. (2009) Thrombotic risk factors in patients with liver cirrhosis: correlation with MELD scoring system and portal vein thrombosis development. J Hepatol 51:682-689

14. La Mura V, Pasarin M, Rodriguez-Vilarrupla A, et al. (2014) Liver sinusoidal endothelial dysfunction after LPS administration: a role for inducible-nitric oxide synthase. J Hepatol 61:1321-1327

15. Yerdel MA, Gunson B, Mirza D, et al. (2000) Portal vein thrombosis in adults undergoing liver transplantation: risk factors, screening, management, and outcome. Transplantation 69:1873-1881

16. Sarin SK, Philips CA, Kamath PS, et al. (2016) Toward a comprehensive new classification of portal vein thrombosis in patients with cirrhosis. Gastroenterology 151(574-577):e573

17. European Association for the Study of the Liver (2016) Electronic address eee. EASL clinical practice guidelines: vascular diseases of the liver. J Hepatol 64:179-202

18. Berzigotti A, Garcia-Criado A, Darnell A, Garcia-Pagan JC (2014) Imaging in clinical decision-making for portal vein thrombosis. Nat Rev Gastroenterol Hepatol 11:308-316

19. Berzigotti A, Piscaglia F (2011) Ultrasound in portal hypertensionpart 1. Ultraschall Med 32:548-568 (quiz 569-571)

20. Berzigotti A, Piscaglia F, Education E, Professional Standards C (2012) Ultrasound in portal hypertension-part 2-and EFSUMB recommendations for the performance and reporting of ultrasound examinations in portal hypertension. Ultraschall Med 33:8-32 (quiz 30-31)

21. Lai L, Brugge WR (2004) Endoscopic ultrasound is a sensitive and specific test to diagnose portal venous system thrombosis (PVST). Am J Gastroenterol 99:40-44

22. Wiersema MJ, Chak A, Kopecky KK, Wiersema LM (1995) Duplex Doppler endosonography in the diagnosis of splenic vein, portal vein, and portosystemic shunt thrombosis. Gastrointest Endosc 42:19-26

23. Claudon M, Dietrich CF, Choi BI, et al. (2013) Guidelines and good clinical practice recommendations for contrast enhanced ultrasound (CEUS) in the liver-update 2012: a WFUMB-EFSUMB initiative in cooperation with representatives of AFSUMB, AIUM, ASUM, FLAUS and ICUS. Ultrasound Med Biol 39:187-210

24. Chen H, Turon F, Hernandez-Gea V, et al. (2016) Nontumoral portal vein thrombosis in patients awaiting liver transplantation. Liver Transpl 22:352-365

25. Piscaglia F, Gianstefani A, Ravaioli M, et al. (2010) Criteria for diagnosing benign portal vein thrombosis in the assessment of patients with cirrhosis and hepatocellular carcinoma for liver transplantation. Liver Transpl 16:658-667

26. Ueno N, Kawamura H, Takahashi H, et al. (2006) Characterization of portal vein thrombus with the use of contrast-enhanced sonography. J Ultrasound Med 25:1147-1152

27. Qi X, Han G, He C, et al. (2012) CT features of non-malignant portal vein thrombosis: a pictorial review. Clin Res Hepatol Gastroenterol 36:561-568 
28. Lee HK, Park SJ, Yi BH, et al. (2008) Portal vein thrombosis: CT features. Abdom Imaging 33:72-79

29. Vilgrain V, Condat B, Bureau C, et al. (2006) Atrophy-hypertrophy complex in patients with cavernous transformation of the portal vein: CT evaluation. Radiology 241:149-155

30. McDonald JS, McDonald RJ, Carter RE, et al. (2014) Risk of intravenous contrast material-mediated acute kidney injury: a propensity score-matched study stratified by baseline-estimated glomerular filtration rate. Radiology 271:65-73

31. Mitchell AM, Kline JA (2007) Contrast nephropathy following computed tomography angiography of the chest for pulmonary embolism in the emergency department. J Thromb Haemost 5:50-54

32. Ascenti G, Sofia C, Silipigni S, et al. (2015) Dual-energy multidetector computed tomography with iodine quantification in the evaluation of portal vein thrombosis: is it possible to discard the unenhanced phase? Can Assoc Radiol J 66:348-355

33. Shah TU, Semelka RC, Voultsinos V, et al. (2006) Accuracy of magnetic resonance imaging for preoperative detection of portal vein thrombosis in liver transplant candidates. Liver Transpl 12:1682-1688

34. Ponziani FR, Zocco MA, Campanale C, et al. (2010) Portal vein thrombosis: insight into physiopathology, diagnosis, and treatment. World J Gastroenterol 16:143-155

35. Martin DR (2008) Nephrogenic systemic fibrosis. Pediatr Radiol 38(Suppl 1):S125-S129

36. Media ACoRCoDaC (2017) ACR manual on contrast media-version 10.3. Reston: American College of Radiology

37. Martin DR, Kalb B, Mittal A, et al. (2017) No incidence of nephrogenic systemic fibrosis after gadobenate dimeglumine administration in patients undergoing dialysis or those with severe chronic kidney disease. Radiology. doi:10.1148/radiol.2017170102

38. Shimada K, Isoda H, Okada T, et al. (2009) Non-contrast-enhanced MR angiography for selective visualization of the hepatic vein and inferior vena cava with true steady-state free-precession sequence and time-spatial labeling inversion pulses: preliminary results. J Magn Reson Imaging 29:474-479

39. Miyazaki M, Isoda H (2011) Non-contrast-enhanced MR angiography of the abdomen. Eur J Radiol 80:9-23

40. Furuta A, Isoda H, Yamashita R, et al. (2014) Non-contrast-enhanced MR portography with balanced steady-state free-precession sequence and time-spatial labeling inversion pulses: comparison of imaging with flow-in and flow-out methods. J Magn Reson Imaging 40:583-587

41. Le Bihan D (1991) Molecular diffusion nuclear magnetic resonance imaging. Magn Reson Q 7:1-30

42. Taouli B, Koh DM (2010) Diffusion-weighted MR imaging of the liver. Radiology 254:47-66

43. Charles-Edwards EM, deSouza NM (2006) Diffusion-weighted magnetic resonance imaging and its application to cancer. Cancer Imaging 6:135-143

44. Ascenti G, Sofia C, Mazziotti S, et al. (2016) Dual-energy CT with iodine quantification in distinguishing between bland and neoplastic portal vein thrombosis in patients with hepatocellular carcinoma. Clin Radiol 71(938):e931-e939

45. Qian LJ, Zhu J, Zhuang ZG, et al. (2012) Differentiation of neoplastic from bland macroscopic portal vein thrombi using dualenergy spectral CT imaging: a pilot study. Eur Radiol 22:2178-2185

46. Catalano OA, Choy G, Zhu A, Hahn PF, Sahani DV (2010) Differentiation of malignant thrombus from bland thrombus of the portal vein in patients with hepatocellular carcinoma: application of diffusion-weighted MR imaging. Radiology 254:154-162

47. Sandrasegaran K, Tahir B, Nutakki K, et al. (2013) Usefulness of conventional MRI sequences and diffusion-weighted imaging in differentiating malignant from benign portal vein thrombus in cirrhotic patients. AJR Am J Roentgenol 201:1211-1219

48. Ahn JH, Yu JS, Cho ES, et al. (2016) Diffusion-weighted mri of malignant versus benign portal vein thrombosis. Korean J Radiol $17: 533-540$
49. Rammohan A, Jeswanth S, Sukumar R, et al. (2013) Percutaneous ultrasound-guided fine-needle aspiration of portal vein thrombi as a diagnostic and staging technique for hepatocellular carcinoma. Abdom Imaging 38:1057-1060

50. Brugge WR, Lee MJ, Kelsey PB, Schapiro RH, Warshaw AL (1996) The use of EUS to diagnose malignant portal venous system invasion by pancreatic cancer. Gastrointest Endosc 43:561-567

51. Berzigotti A, Abraldes JG, Tandon P, et al. (2010) Ultrasonographic evaluation of liver surface and transient elastography in clinically doubtful cirrhosis. J Hepatol 52:846-853

52. Smith AD, Branch CR, Zand K, et al. (2016) Liver surface nodularity quantification from routine $\mathrm{CT}$ images as a biomarker for detection and evaluation of cirrhosis. Radiology 280:771-781

53. Friedrich-Rust M, Ong MF, Martens S, et al. (2008) Performance of transient elastography for the staging of liver fibrosis: a metaanalysis. Gastroenterology 134:960-974

54. Berzigotti A (2017) Non invasive evaluation of portal hypertension using ultrasound elastography. J Hepatol 67:399-411

55. Delgado MG, Seijo S, Yepes I, et al. (2012) Efficacy and safety of anticoagulation on patients with cirrhosis and portal vein thrombosis. Clin Gastroenterol Hepatol 10:776-783

56. Arora A, Bansal K, Sureka B, Patidar Y (2015) Not to forget portal cavernoma cholangiopathy: the great mimicker!. Abdom Imaging 40:1510-1511

57. Llop E, de Juan C, Seijo S, et al. (2011) Portal cholangiopathy: radiological classification and natural history. Gut 60:853-860

58. Sharma M, Rameshbabu CS (2014) Portal cavernoma cholangiopathy: an endoscopic ultrasound based imaging approach. J Clin Exp Hepatol 4:S53-S61

59. Intagliata NM, Maitland H, Caldwell SH (2016) Direct oral anticoagulants in cirrhosis. Curr Treat Options Gastroenterol $14: 247-256$

60. De Gottardi A, Trebicka J, Klinger C, et al. (2017) Antithrombotic treatment with direct-acting oral anticoagulants in patients with splanchnic vein thrombosis and cirrhosis. Liver Int 37:694-699

61. Chamarthy MR, Anderson ME, Pillai AK, Kalva SP (2016) Thrombolysis and transjugular intrahepatic portosystemic shunt creation for acute and subacute portal vein thrombosis. Tech Vasc Interv Radiol 19:42-51

62. Cherukuri R, Haskal ZJ, Naji A, Shaked A (1998) Percutaneous thrombolysis and stent placement for the treatment of portal vein thrombosis after liver transplantation: long-term follow-up. Transplantation 65:1124-1126

63. Uflacker R (1997) Mechanical thrombectomy in acute and subacute thrombosis with use of the Amplatz device: arterial and venous applications. J Vasc Interv Radiol 8:923-932

64. Ferro C, Rossi UG, Bovio G, Dahamane M, Centanaro M (2007) Transjugular intrahepatic portosystemic shunt, mechanical aspiration thrombectomy, and direct thrombolysis in the treatment of acute portal and superior mesenteric vein thrombosis. Cardiovasc Intervent Radiol 30:1070-1074

65. Uflacker R (2003) Applications of percutaneous mechanical thrombectomy in transjugular intrahepatic portosystemic shunt and portal vein thrombosis. Tech Vasc Interv Radiol 6:59-69

66. Bilbao JI, Elorz M, Vivas I, et al. (2004) Transjugular intrahepatic portosystemic shunt (TIPS) in the treatment of venous symptomatic chronic portal thrombosis in non-cirrhotic patients. Cardiovasc Intervent Radiol 27:474-480

67. Habib A, Desai K, Hickey R, et al. (2015) Portal vein recanalization-transjugularintrahepatic portosystemic shunt using the transsplenic approach to achieve transplant candidacy in patients with chronic portal vein thrombosis. J Vasc Interv Radiol 26:499-506

68. Wang Z, Jiang MS, Zhang HL, et al. (2016) Is Post-TIPS anticoagulation therapy necessary in patients with cirrhosis and portal vein thrombosis? A Randomized Controlled Trial. Radiology 279:943-951 